MONTES O; DIÁNEZ F; CAMACHO F. 2014. Doses of caffeine on the development and performance of pepper crops under greenhouse. Horticultura Brasileira 32: 398-403. DOI - http://dx.doi.org/10.1590/S0102-053620140000400005

\title{
Doses of caffeine on the development and performance of pepper crops under greenhouse
}

\author{
Óscar Montes; Fernando Diánez; Francisco Camacho
}

University of Almeria, 04120 Almeria-Spain; osmonza@hotmail.com; fdianez@ual.es; fcamacho@ual.es (author for correspondence)

\begin{abstract}
A number of experiments on plant organs demonstrated that caffeine acts as a growth stimulator. In this study we aimed to determine the influence of caffeine on development of pepper crops under stress in the autumn-winter cycle, as peppers stop growing and developing in November and January. The experiments were conducted to determine how caffeine applied to the soil by fertigation, with doses of $2.25 \mu \mathrm{M}$ and $9.00 \mu \mathrm{M}$ for $\mathrm{T}_{1}$ and $\mathrm{T}_{2}$, respectively, compared to the control crop, affect the morphological development and performance of the pepper crops during two crop cycles (20082009 and 2009-2010 in autumn-winter). Throughout the whole vegetative plant cycle, performance parameters of fruit quality and the influence on the morphological development were obtained on different dates. With respect to the remainder of the morphological and production parameters, no significant differences were discovered in either of the evaluated cycles, thus leading to the conclusion that the two doses of caffeine, $2.25 \mu \mathrm{M}$ and $9.00 \mu \mathrm{M}$, did not have any stimulant effect on the development and performance of the pepper crop during both cycles. Total yields were unaffected by either treatment, $4.89,4.90$ and $4.88 \mathrm{~kg} / \mathrm{m}^{2}$ for the first assay and 5.28 , 5.23 and 5.28 for the second, for treatments control and caffeine doses $2.25 \mu \mathrm{M}$ and $9.00 \mu \mathrm{M}$ respectively.
\end{abstract}

Keywords: Capsicum annuum, 1,3,7 trimethylxanthine, alkaloid, growth inducer, allelopathic.

\section{RESUMEN}

Efecto de dosis de cafeína sobre el desarrollo y el comportamiento de cultivos de pimiento en invernadero

Un gran número de experimentos realizados sobre distintos órganos vegetales han puesto de manifiesto que la cafeína actúa como estimulador del crecimiento vegetal. El objetivo del presente estudio, fue determinar la influencia de la cafeína sobre el desarrollo de cultivos de pimiento en condiciones de estrés durante el ciclo de otoño-invierno, en el cual este cultivo detiene su crecimiento y desarrollo durante los meses de Noviembre a Enero. Se llevaron a cabo experimentos para determinar los efectos de la cafeína sobre el desarrollo morfológico y el rendimiento en cultivo de pimiento en invernadero, durante dos ciclos de cultivo 2008/2009 y 2009/2010 (ciclo de otoño-inverno). La aplicación se realizó al suelo en fertirrigación con dosis constantes de $2,25 \mu \mathrm{M}$ y 9,00 $\mu \mathrm{M}$ para $\mathrm{T} 1 \mathrm{y}$ T2 respectivamente, comparándolo con el cultivo control. Durante todo el ciclo se evaluaron para los distintos momentos de recolección parámetros de rendimiento y calidad de fruto, así como la influencia en el desarrollo morfológico del mismo evaluada al finalizar el cultivo. Los resultados concluyen (para ambos ciclos evaluados) que la cafeína no tiene efecto estimulante sobre la cantidad o calidad de la cosecha, ni sobre los parámetros morfológicos asociados al vigor de la planta, no existiendo diferencias significativas para la mayoría de los parámetros determinados a las dosis evaluadas de $2,25 \mu \mathrm{M}$ y $9,00 \mu \mathrm{M}$ aplicadas de forma continuada. Los rendimientos finales no fueron influenciados pelos tratamiento, 4,89, 4,90 y $4,88 \mathrm{~kg} / \mathrm{m}^{2}$ en el primer ensayo y de 5,28, 5,23 y 5,28 en el segundo, para los tratamientos testigo y dosis de cafeína de $2,25 \mu \mathrm{M}$ y $9,00 \mu \mathrm{M}$ respectivamente.

Palabras clave: Capsicum annuum, 1,3,7 trimetilxantina, alcaloide, inductor del crecimiento, alelopático.

(Recebido para publicação em 16 de outubro de 2013; aceito em 28 de agosto de 2014) (Received on October 16, 2013; accepted on August 28, 2014)

$\mathrm{P}$ ants synthesise a wide range of secondary metabolites with diverse chemical structures, such as alkaloids, flavonoids, phenols, steroids and terpenoids (Hadacek, 2002). These compounds are believed to play major roles in the adaptation of plants to the environment (Croteau et al., 2000; Taiz \& Zeiger, 2002).

Alkaloids are a group of nitrogen compounds that are present in plants (Wink, 2004). Caffeine (1,3,7 trimethylxanthine) is an alkaloid derived from purine, which is naturally found in approximately 100 plant species (Ashihara, 2006).

Several studies have highlighted the role that caffeine plays as a plant growth inducer, while other studies have investigated its role as a plant growth reducer; caffeine is connected with allelopathic effects, including inter and intraspecific effects (Singh et al., 2001; Hesse, 2002; Uefuji et al., 2003; Wink, 2004). Additionally, caffeine has proved to be an effective pesticide (Mathavan et al., 1985; Hollingsworth et al., 2002; Kim et al., 2006).

Caffeine is similar in structure to cytokinins. The intact purine ring 
is essential for cytokinin activity in plants (Matsubara, 1990). Cytokinins in Raphanus sativus cotyledons are deactivated by caffeine (Tao et al., 1991). Cytokinins produce different biological activities in the plant, including stimulation of cell division, promotion of seed germination, growth of cotyledons and leaves, development of secondary stems, elongation of both stems and roots (Hitoshi, 2006), and inhibition of the chlorophyll degradation (Mok, 1994). Caffeine has stimulant or inhibiting effects, which promote stomatal opening in the epidermis of Vicia faba (Morsucci et al., 1991). Internodes can become etiolated in Glycine max due to caffeine and chlorophyll in Cucumis sativus can be increased (Vitória \& Mazzafera, 1998).

Batish et al. (2008) applied caffeine in concentrations from 50 to 2000 $\mu \mathrm{M}$ to Phaseolus aureus hypocotyls cuttings and number and length of roots was reduced. Caffeine concentrations between 10,000 and 25,000 $\mu \mathrm{M}$ reduced numbers of root cells in Pisum sativum seedlings (Curlango-Rivera et al., 2010). Concentration of $5,154 \mu \mathrm{M}$ caffeine increased root mitosis in meristematic cells of Phaseolus vulgaris (Truta et al., 2011). Concentration of $2500 \mu \mathrm{M}$ caffeine inhibits root mitosis in Zea mays (Anaya et al., 2002) Oriza sativa (Smyth, 1992). Caffeine concentrations between 2,575, and 38,660 $\mu \mathrm{M}$ increase the mean height in plants of Capsicum annum (Kumar \& Tripathi, 2004).

Greenhouse crops represent a large proportion of vegetable production. Pepper is a key vegetable along the Mediterranean coast. Pepper (Capsicum annuum) is the second most important specie, after tomato, produced in Almería, Spain, where fruiting occurs from October to March, and Murcia, where the harvest is from March to July. In Almeria, the price of peppers usually peaks during the winter and early spring. The highest pepper prices in Murcia and Alicante occur between March and April for green peppers and between April and May for red peppers. During the production cycle, plants are under abiotic stress because production facilities have no climate control; this stress is related to thermal fluctuations (excess heat or cold) which influence development. This study aimed to determine the influence of caffeine on development of pepper crops under stress in the autumn-winter cycle, as peppers stop growing and developing in November and January, causing a drop in the production, because of low temperatures and lack of light.

\section{MATERIAL AND METHODS}

The experiment was conducted during the autumn-winter seasons of 2008-2009 and 2009-2010 at the experimental foundation UALANECOOP, situated in the town of Retamar within the municipality of Almeria ( $2^{0} 17^{\prime} 08^{\prime \prime} \mathrm{W}, 36^{0} 51^{\prime} 77^{\prime \prime} \mathrm{N}$, $88 \mathrm{~m}$ above sea level). The climate is Mediterranean semi-arid with below $300 \mathrm{~mm} /$ year of precipitation yearly average temperature ranges from 15 to $21^{\circ} \mathrm{C}$. The winter is mild and contains significant thermal fluctuations. The average temperature during January and February is approximately $6^{\circ} \mathrm{C}$.

The experiment was performed in an area of the experimental station oriented east-west direction, in a greenhouse with a cultivable area of $1,695 \mathrm{~m}^{2}$. The greenhouse has a metal frame covered by a gable roof. The facility includes wing speed, temperature and rain sensors for the automatic opening and closing of the zenithal windows. The roof was made of 800-gaugethick polyethylene. The peppers were transplanted on $22^{\text {th }}$ July, 2008 and on $22^{\text {th }}$ July, 2009, respectively.

The cultivar used was Misano a "California-type" red-ripe pepper. It is recommended as an extra-early and early crop in Almeria. The greenhouse had two irrigation sectors containing rot fertilizers, which had a capacity of $40 \mathrm{~L}$ and ran simultaneously and independently from each other. Caffeine doses were deposited in the fertilizers that were assigned to each treatment. Fertigation was conducted three times per week. The amount of water supplied was established by the needs of the crop and the climactic conditions in the greenhouse. The EC was adjusted to $1.8 \mathrm{dS} / \mathrm{m}$.
The design was a randomised complete block, with 3 treatments and 4 replications. The treatments were $\mathrm{T}_{0}=$ control, consisting of application of an ideal solution as the balanced fertilizer (10 $\mathrm{mmol} / \mathrm{L}$ of $\mathrm{NO}_{3}^{-} ; 2 \mathrm{mmol} / \mathrm{L}_{2}$ of $\mathrm{H}_{2} \mathrm{PO}_{4}^{-} ; 2$ $\mathrm{mmol} / \mathrm{L}$ of $\mathrm{SO}_{4}^{-2} ; 0.5 \mathrm{mmol} / \mathrm{L}^{-2} \mathrm{HCO}_{3}^{-}$; $1.6 \mathrm{mmol} / \mathrm{L}$ of $\mathrm{NH}_{4}^{+} ; 6 \mathrm{mmol} / \mathrm{L}$ of $\mathrm{K}^{+}$; $4 \mathrm{mmol} / \mathrm{L}$ of $\mathrm{Ca}^{+2}$ and $2 \mathrm{mmol} / \mathrm{L}$ of $\left.\mathrm{Mg}^{+2}\right) ; \mathrm{T}_{1}$ and $\mathrm{T}_{2}$ were the same ideal solution with a concentration of 2.25 and $9.00 \mu \mathrm{M}$ of caffeine respectively. Caffeine concentrations were applied in a consistent way through frequent irrigation (3 irrigations per week) until a caffeine accumulation of 378 (T1) and 1,512 $\mu \mathrm{M}$ (T2) or 323 (T1) and 1,292 $\mu \mathrm{M}$ (T2) per pepper plant was achieved at the end of each evaluated cycle.

Plants were established in double lines separated by $0.83 \mathrm{~m}$. In row plant spacing was $0.50 \mathrm{~m}$ resulting in a planting density of 2.3 plants $/ \mathrm{m}^{2}$. Four rows were used for samples of each plot, resulting in 16 rows per treatment in total.

At the end of the crop cycle of each plot, 10 plants were obtained at random and various measurements taken (Figure 1). The measured parameters were: stem length (SL), distance to the first bifurcation from the root collar; branch length (BL), distance from the fork to the apex of each branch, a number was assigned to each branch (branch 1 and branch 2); diameter at the stem base (DSB); diameter of the stem base measured above the root-collar; diameter of the stem fork (FSD): diameter of the main stem below the fork, and diameter of branches 1 and 2 (FBD and SBD): the diameter of each branch was measured half way between the fourth internode.

The evaluation of production was made as follows: During sorting, marketable fruits were identified and weighed on a high-precision balance, model PM III (Gram Precision S.L. Spain), The marketable yield, fruit average weight and number of fruits $/ \mathrm{m}^{2}$ were obtained. The equatorial and polar diameter of 15 randomly obtained fruits was determined from each replication on each harvest.

Data were analyzed using the Statgraphics 5.1 statistical package for Windows (Statgraphics, Warrenton, VA, 
USA), an analysis of variance (ANOVA) was performed. Significance of differences among treatments was tested using the least significant difference
(LSD) method. Differences were judged significant at $\mathrm{p}<0.05$ according to the F-test. The F-protected LSD values were calculated at 0.05 probability levels.

\section{RESULTS AND DISCUSSION}

Treatment did not affect most of the morphological studied parameters like

Table 1. Morphological parameters of the pepper cv. Misano. Data recorded at the end of the harvest (parámetros morfológicos del pimiento cv. Misano. Datos registrados al final de cosecha). Almería (Spain), Foundation UAL, 2008-2010.

\begin{tabular}{|c|c|c|c|c|c|c|c|c|c|}
\hline Treatment & \multicolumn{9}{|c|}{ Variables } \\
\hline \multirow{2}{*}{ 2008-09 } & SL & FBL & SBL & ILFB & ILSB & Ø DSB & Ø FSD & Ø FBD & Ø SBD \\
\hline & \multicolumn{5}{|c|}{ (cm) } & \multicolumn{4}{|c|}{$(\mathbf{m m})$} \\
\hline $\mathrm{T}_{0}$ & $40.5 \mathrm{a}$ & $96.0 \mathrm{a}$ & $92.2 \mathrm{a}$ & $7.3 \mathrm{a}$ & $7.8 \mathrm{a}$ & $18.19 \mathrm{a}$ & $16.83 \mathrm{a}$ & $11.17 \mathrm{a}$ & $10.94 \mathrm{a}$ \\
\hline $\mathrm{T}_{1}$ & $39.3 \mathrm{a}$ & $93.6 \mathrm{a}$ & $96.6 \mathrm{a}$ & $7.4 \mathrm{a}$ & $7.5 \mathrm{a}$ & $18.38 \mathrm{a}$ & $17.31 \mathrm{a}$ & $11.71 \mathrm{a}$ & $12.32 \mathrm{a}$ \\
\hline $\mathrm{T}_{2}$ & $39.5 \mathrm{a}$ & $95.1 \mathrm{a}$ & $93.9 \mathrm{a}$ & $7.3 \mathrm{a}$ & $7.6 \mathrm{a}$ & $18.87 \mathrm{a}$ & $16.89 \mathrm{a}$ & $11.57 \mathrm{a}$ & $12.10 \mathrm{a}$ \\
\hline P-value & 0.15 & 0.78 & 0.75 & 0.26 & 0.13 & 0.17 & 0.49 & 0.26 & 0.52 \\
\hline \multicolumn{10}{|l|}{ 2009-10 } \\
\hline $\mathrm{T}_{0}$ & $41.20 \mathrm{a}$ & $107.2 \mathrm{a}$ & $121.10 \mathrm{a}$ & $7.32 \mathrm{a}$ & $7.70 \mathrm{a}$ & $20.75 \mathrm{a}$ & $17.17 \mathrm{a}$ & $11.92 \mathrm{a}$ & $11.90 \mathrm{a}$ \\
\hline $\mathrm{T}_{1}$ & $39.60 \mathrm{a}$ & $105.7 \mathrm{a}$ & $117.25 \mathrm{a}$ & $7.22 \mathrm{a}$ & $7.12 \mathrm{a}$ & 20.99 a & $17.52 \mathrm{a}$ & $12.48 \mathrm{a}$ & $12.35 \mathrm{a}$ \\
\hline $\mathrm{T}_{2}$ & $40.55 \mathrm{a}$ & $118.1 \mathrm{~b}$ & $111.65 \mathrm{a}$ & $7.49 \mathrm{a}$ & $7.61 \mathrm{a}$ & $20.52 \mathrm{a}$ & $16.71 \mathrm{a}$ & $12.39 \mathrm{a}$ & $12.02 \mathrm{a}$ \\
\hline P-value & 0.35 & 0.03 & 0.72 & 0.16 & 0.56 & 0.76 & 0.48 & 0.34 & 0.26 \\
\hline
\end{tabular}

Different letters within a row represent significant differences at $p<0.05$. Stem length (SL), diameter at the stem base (DSB), diameter of the stem fork (FSD), first and second branch length (FBL and SBL), diameter of first and second branch (FBD and SBD), average internode length of first and second branch (ILFB and ILSB) (letras distintas dentro de una fila representan diferencias significativas a $p<0,05$. Longitud del tallo (SL), diámetro en la base del tallo (DSB), diámetro del tallo en la bifurcación (FSD), longitud de primera y segunda rama (FBL y SBL), diámetro de la primera y segunda rama (FBD y SBD), longitud media entre nudos de la primera y segunda rama (ILFB y ILSB).

Table 2. Performance parameter for total yield $\left(\mathrm{kg} / \mathrm{m}^{2}\right)$ and number of fruits $/ \mathrm{m}^{2}$ for the pepper cv. Misano (parámetros de rendimiento total $\left(\mathrm{kg} / \mathrm{m}^{2}\right)$ y número de frutos $/ \mathrm{m}^{2}$ para el pimiento cv. Misano). Almería (Spain), Foundation UAL, 2008-2010.

\begin{tabular}{|c|c|c|c|c|c|c|c|c|c|c|c|c|}
\hline Treatment & & & & & & & AT & & & & & \\
\hline 2008-09* & 78 & 84 & 91 & 98 & 105 & 112 & 119 & 131 & 138 & 153 & 166 & 180 \\
\hline $\mathrm{T}_{0}$ & $0.19 \mathrm{a}$ & $0.66 \mathrm{a}$ & $1.08 \mathrm{a}$ & $1.28 \mathrm{a}$ & $1.80 \mathrm{a}$ & $2.25 \mathrm{a}$ & $2.58 \mathrm{a}$ & $2.97 \mathrm{a}$ & $3.13 \mathrm{a}$ & $3.90 \mathrm{a}$ & $4.39 \mathrm{a}$ & $4.89 \mathrm{a}$ \\
\hline $\mathrm{T}_{1}$ & $0.21 \mathrm{a}$ & $0.71 \mathrm{a}$ & $1.12 \mathrm{a}$ & $1.36 \mathrm{a}$ & $1.89 \mathrm{a}$ & $2.33 \mathrm{a}$ & $2.67 \mathrm{a}$ & $3.02 \mathrm{a}$ & $3.20 \mathrm{a}$ & $4.02 \mathrm{a}$ & $4.39 \mathrm{a}$ & $4.90 \mathrm{a}$ \\
\hline $\mathrm{T}_{2}$ & $0.18 \mathrm{a}$ & $0.66 \mathrm{a}$ & $1.10 \mathrm{a}$ & $1.32 \mathrm{a}$ & $1.88 \mathrm{a}$ & $2.35 \mathrm{a}$ & $2.69 \mathrm{a}$ & $3.02 \mathrm{a}$ & $3.21 \mathrm{a}$ & $3.97 \mathrm{a}$ & $4.31 \mathrm{a}$ & $4.88 \mathrm{a}$ \\
\hline P-value & 0.76 & 0.82 & 0.95 & 0.21 & 0.74 & 0.82 & 0.78 & 0.94 & 0.88 & 0.80 & 0.90 & 0.99 \\
\hline 2009-10* & 87 & 94 & 101 & 108 & 115 & 122 & 129 & 136 & 143 & 150 & 162 & 175 \\
\hline $\mathrm{T}_{0}$ & $0.23 \mathrm{a}$ & $0.54 \mathrm{a}$ & $0.94 \mathrm{a}$ & $1.58 \mathrm{a}$ & $2.18 \mathrm{a}$ & $2.84 \mathrm{a}$ & $3.43 \mathrm{a}$ & $3.65 \mathrm{a}$ & $3.77 \mathrm{a}$ & $4.19 \mathrm{a}$ & $4.68 \mathrm{a}$ & $5.28 \mathrm{a}$ \\
\hline $\mathrm{T}_{1}$ & $0.17 \mathrm{a}$ & $0.51 \mathrm{a}$ & $0.90 \mathrm{a}$ & $1.47 \mathrm{a}$ & $2.12 \mathrm{a}$ & $2.78 \mathrm{a}$ & $3.31 \mathrm{a}$ & $3.52 \mathrm{a}$ & $3.63 \mathrm{a}$ & $4.10 \mathrm{a}$ & $4.55 \mathrm{a}$ & $5.23 \mathrm{a}$ \\
\hline $\mathrm{T}_{2}$ & $0.22 \mathrm{a}$ & $0.52 \mathrm{a}$ & $0.89 \mathrm{a}$ & $1.46 \mathrm{a}$ & $2.22 \mathrm{a}$ & $2.98 \mathrm{a}$ & $3.39 \mathrm{a}$ & $3.59 \mathrm{a}$ & $3.67 \mathrm{a}$ & $4.13 \mathrm{a}$ & $4.60 \mathrm{a}$ & $5.28 \mathrm{a}$ \\
\hline P-value & 0.56 & 0.94 & 0.94 & 0.68 & 0.76 & 0.28 & 0.70 & 0.68 & 0.71 & 0.91 & 0.87 & 0.98 \\
\hline $2008-09 * *$ & 78 & 84 & 91 & 98 & 105 & 112 & 119 & 131 & 138 & 153 & 166 & 180 \\
\hline $\mathrm{T}_{0}$ & $1.2 \mathrm{a}$ & $4.0 \mathrm{a}$ & $6.2 \mathrm{a}$ & $7.4 \mathrm{a}$ & $10.0 \mathrm{a}$ & $12.3 \mathrm{a}$ & $14.1 \mathrm{a}$ & $16.0 \mathrm{a}$ & $16.8 \mathrm{a}$ & $20.8 \mathrm{a}$ & $23.6 \mathrm{a}$ & $27.0 \mathrm{a}$ \\
\hline $\mathrm{T}_{1}$ & $1.4 \mathrm{a}$ & $4.4 \mathrm{a}$ & $6.7 \mathrm{a}$ & $8.0 \mathrm{a}$ & $10.9 \mathrm{a}$ & $13.3 \mathrm{a}$ & $15.1 \mathrm{a}$ & $16.9 \mathrm{a}$ & $17.7 \mathrm{a}$ & $21.9 \mathrm{a}$ & $24.1 \mathrm{a}$ & $28.0 \mathrm{a}$ \\
\hline $\mathrm{T}_{2}$ & $1.2 \mathrm{a}$ & $4.1 \mathrm{a}$ & $6.5 \mathrm{a}$ & $7.7 \mathrm{a}$ & $10.6 \mathrm{a}$ & $13.0 \mathrm{a}$ & $14.8 \mathrm{a}$ & $16.6 \mathrm{a}$ & $17.9 \mathrm{a}$ & $21.4 \mathrm{a}$ & $23.3 \mathrm{a}$ & $27.5 \mathrm{a}$ \\
\hline P-value & 0.66 & 0.88 & 0.85 & 0.59 & 0.58 & 0.62 & 0.61 & 0.72 & 0.68 & 0.61 & 0.83 & 0.79 \\
\hline $2009-10 * *$ & 87 & 94 & 101 & 108 & 115 & 122 & 129 & 136 & 143 & 150 & 162 & 175 \\
\hline $\mathrm{T}_{0}$ & $1.3 \mathrm{a}$ & $3.0 \mathrm{a}$ & $5.1 \mathrm{a}$ & $8.0 \mathrm{a}$ & $10.9 \mathrm{a}$ & $14.1 \mathrm{a}$ & $17.0 \mathrm{a}$ & $18.1 \mathrm{a}$ & $18.6 \mathrm{a}$ & $20.7 \mathrm{a}$ & $23.4 \mathrm{a}$ & $26.7 \mathrm{a}$ \\
\hline $\mathrm{T}_{1}$ & $1.0 \mathrm{a}$ & $2.9 \mathrm{a}$ & $5.0 \mathrm{a}$ & $7.7 \mathrm{a}$ & $10.8 \mathrm{a}$ & $14.2 \mathrm{a}$ & $16.7 \mathrm{a}$ & $17.7 \mathrm{a}$ & $18.2 \mathrm{a}$ & $20.5 \mathrm{a}$ & $22.9 \mathrm{a}$ & $26.5 \mathrm{a}$ \\
\hline $\mathrm{T}_{2}$ & $1.3 \mathrm{a}$ & $2.9 \mathrm{a}$ & $4.8 \mathrm{a}$ & $7.6 \mathrm{a}$ & $11.3 \mathrm{a}$ & $15.0 \mathrm{a}$ & $17.1 \mathrm{a}$ & $18.1 \mathrm{a}$ & $18.5 \mathrm{a}$ & $20.7 \mathrm{a}$ & $23.2 \mathrm{a}$ & $26.5 \mathrm{a}$ \\
\hline P-value & 0.55 & 0.79 & 0.86 & 0.79 & 0.79 & 0.74 & 0.78 & 0.85 & 0.33 & 0.35 & 0.35 & 0.43 \\
\hline
\end{tabular}

Different letters within a row represent significant differences at $\mathrm{p}<0.05$; DAT $=$ days after transplant (letras distintas dentro de una fila representan diferencias significativas a $\mathrm{p}<0,05$; DAT $=$ días después del trasplante); *values corresponding to total yield of fruits $(\mathrm{kg} / \mathrm{m} 2)\{\mathrm{valores}$ correspondientes a rendimiento total de frutas $(\mathrm{kg} / \mathrm{m} 2)\} ; * *$ values corresponding to number of fruits $/ \mathrm{m} 2$ (valores correspondientes a número de frutas $/ \mathrm{m} 2$ ). 
stem length (SL), stem base diameter (DSB), fork stem diameter (FSD), second branch length (SBL), first branch diameter (FBD), second branch diameter (SBD) and internode length of the first branch (ILFB) (Table 1). Just significant differences were observed during the second cycle for first branch length (FBL). $\mathrm{T}_{2}(9.00 \mu \mathrm{M}$ of caffeine) had the highest FBL at a length of 118.10 $\mathrm{cm}$, while $\mathrm{T}_{1}(2.25 \mu \mathrm{M}$ of caffeine $)$ had the shortest FBL $105.7 \mathrm{~cm}$. There were no significant differences in FBL observed during the first cycle (season 2008/09) (Table 1).

Treatment did not affect most of the production-studied parameters like total yield, number of marketable fruits/ $\mathrm{m}^{2}$ (Table 2) and fruit's average weight (Table 3). During the first and second cycle (2008/09 and 2009/10), significant differences in the equatorial and polar diameters of the fruit were found, but there were not a clear relationship between treatment and diameters (Table 4). Montes et al. (2013) did not find differences in watermelon production

Table 3. Average weight of fruit ( $\mathrm{g}$ ) for the pepper cv. Misano, over time (peso medio del fruto ( $\mathrm{g}$ ) para el pimiento cv. Misano, con respecto al tiempo). Almería (Spain), Foundation UAL, 2008-2010.

\begin{tabular}{lcccccccccccc}
\hline Treatment & \multicolumn{10}{c}{ DAT } \\
\hline $\mathbf{2 0 0 8 - 0 9}$ & $\mathbf{7 8}$ & $\mathbf{8 4}$ & $\mathbf{9 1}$ & $\mathbf{9 8}$ & $\mathbf{1 0 5}$ & $\mathbf{1 1 2}$ & $\mathbf{1 1 9}$ & $\mathbf{1 3 1}$ & $\mathbf{1 3 8}$ & $\mathbf{1 5 3}$ & $\mathbf{1 6 6}$ & $\mathbf{1 8 0}$ \\
\hline $\mathrm{T}_{0}$ & $159 \mathrm{a}$ & $165 \mathrm{a}$ & $196 \mathrm{a}$ & $184 \mathrm{a}$ & $193 \mathrm{a}$ & $198 \mathrm{a}$ & $186 \mathrm{a}$ & $200 \mathrm{a}$ & $211 \mathrm{a}$ & $192 \mathrm{a}$ & $174 \mathrm{a}$ & $153 \mathrm{a}$ \\
$\mathrm{T}_{1}$ & $153 \mathrm{a}$ & $166 \mathrm{a}$ & $181 \mathrm{a}$ & $171 \mathrm{a}$ & $184 \mathrm{a}$ & $188 \mathrm{a}$ & $189 \mathrm{a}$ & $195 \mathrm{a}$ & $222 \mathrm{a}$ & $195 \mathrm{a}$ & $173 \mathrm{a}$ & $132 \mathrm{a}$ \\
$\mathrm{T}_{2}$ & $155 \mathrm{a}$ & $163 \mathrm{a}$ & $189 \mathrm{a}$ & $180 \mathrm{a}$ & $198 \mathrm{a}$ & $194 \mathrm{a}$ & $192 \mathrm{a}$ & $193 \mathrm{a}$ & $208 \mathrm{a}$ & $192 \mathrm{a}$ & $180 \mathrm{a}$ & $138 \mathrm{a}$ \\
$\mathrm{p}$-value & 0.20 & 0.89 & 0.17 & 0.15 & 0.57 & 0.51 & 0.84 & 0.59 & 0.15 & 0.78 & 0.82 & 0.54 \\
\hline $\mathbf{2 0 0 9 - 1 0}$ & $\mathbf{8 7}$ & $\mathbf{9 4}$ & $\mathbf{1 0 1}$ & $\mathbf{1 0 8}$ & $\mathbf{1 1 5}$ & $\mathbf{1 2 2}$ & $\mathbf{1 2 9}$ & $\mathbf{1 3 6}$ & $\mathbf{1 4 3}$ & $\mathbf{1 5 0}$ & $\mathbf{1 6 2}$ & $\mathbf{1 7 5}$ \\
\hline $\mathrm{T}_{0}$ & $175 \mathrm{a}$ & $179 \mathrm{a}$ & $197 \mathrm{a}$ & $210 \mathrm{a}$ & $211 \mathrm{a}$ & $207 \mathrm{a}$ & $209 \mathrm{a}$ & $204 \mathrm{a}$ & $215 \mathrm{a}$ & $197 \mathrm{a}$ & $184 \mathrm{a}$ & $194 \mathrm{a}$ \\
$\mathrm{T}_{1}$ & $167 \mathrm{a}$ & $177 \mathrm{a}$ & $190 \mathrm{a}$ & $208 \mathrm{a}$ & $204 \mathrm{a}$ & $202 \mathrm{a}$ & $213 \mathrm{a}$ & $199 \mathrm{a}$ & $207 \mathrm{a}$ & $212 \mathrm{a}$ & $187 \mathrm{a}$ & $192 \mathrm{a}$ \\
$\mathrm{T}_{2}$ & $170 \mathrm{a}$ & $179 \mathrm{a}$ & $196 \mathrm{a}$ & $206 \mathrm{a}$ & $205 \mathrm{a}$ & $201 \mathrm{a}$ & $204 \mathrm{a}$ & $197 \mathrm{a}$ & $205 \mathrm{a}$ & $206 \mathrm{a}$ & $187 \mathrm{a}$ & $203 \mathrm{a}$ \\
\hline $\mathrm{p}$-value & 0.71 & 0.99 & 0.65 & 0.94 & 0.52 & 0.96 & 0.64 & 0.85 & 0.44 & 0.54 & 0.93 & 0.72 \\
\hline
\end{tabular}

Different letters within a row represent significant differences at $P<0.05^{*}$ DAT (days after transplant) (Letras distintas dentro de una fila representan diferencias significativas a $\mathrm{P}<0,05 *$ DAT (días después del trasplante).

Table 4. Equatorial diameter of fruit and polar diameter $(\mathrm{mm})$ for the pepper cv. Misano, over time (diámetro ecuatorial y diámetro polar (mm) del fruto para el pimiento cv. Misano, con respecto al tiempo). Almería (Spain), Foundation UAL, 2008-2010.

\begin{tabular}{|c|c|c|c|c|c|c|c|c|c|c|c|c|}
\hline Treatment & & & & & & & AT & & & & & \\
\hline 2008-09* & 78 & 84 & 91 & 98 & 105 & 112 & 119 & 131 & 138 & 153 & 166 & 180 \\
\hline $\mathrm{T}_{0}$ & $83.1 \mathrm{a}$ & $81.4 \mathrm{a}$ & $86.3 \mathrm{~b}$ & $84.5 \mathrm{a}$ & $86.6 \mathrm{a}$ & $86.4 \mathrm{a}$ & $87.7 \mathrm{a}$ & $88.4 \mathrm{a}$ & $89.8 \mathrm{a}$ & $88.2 \mathrm{a}$ & $94.7 \mathrm{a}$ & $96.6 \mathrm{a}$ \\
\hline $\mathrm{T}_{1}$ & $82.9 \mathrm{a}$ & $80.4 \mathrm{a}$ & $82.7 \mathrm{a}$ & $85.6 \mathrm{a}$ & $87.4 \mathrm{a}$ & $85.8 \mathrm{a}$ & $87.1 \mathrm{a}$ & $88.0 \mathrm{a}$ & $90.2 \mathrm{a}$ & $86.5 \mathrm{a}$ & $93.8 \mathrm{a}$ & $96.4 \mathrm{a}$ \\
\hline $\mathrm{T}_{2}$ & $87.3 \mathrm{a}$ & $81.5 \mathrm{a}$ & $84.3 \mathrm{ab}$ & $85.5 \mathrm{a}$ & $86.0 \mathrm{a}$ & $86.4 \mathrm{a}$ & $88.0 \mathrm{a}$ & $88.0 \mathrm{a}$ & $91.2 \mathrm{a}$ & $89.1 \mathrm{a}$ & $95.3 \mathrm{a}$ & $97.6 \mathrm{a}$ \\
\hline P-value & 0.35 & 0.73 & 0.04 & 0.65 & 0.20 & 0.92 & 0.66 & 0.93 & 0.35 & 0.36 & 0.38 & 0.74 \\
\hline 2009-10* & 87 & 94 & 101 & 108 & 115 & 122 & 129 & 136 & 143 & 150 & 162 & 175 \\
\hline $\mathrm{T}_{0}$ & $78.2 \mathrm{a}$ & $82.9 \mathrm{a}$ & $84.9 \mathrm{~b}$ & $83.4 \mathrm{a}$ & $87.3 \mathrm{a}$ & $86.5 \mathrm{a}$ & $85.2 \mathrm{a}$ & $84.9 \mathrm{a}$ & $88.0 \mathrm{a}$ & $89.8 \mathrm{a}$ & $86.2 \mathrm{a}$ & $89.1 \mathrm{a}$ \\
\hline $\mathrm{T}_{1}$ & $77.2 \mathrm{a}$ & $87.7 \mathrm{a}$ & $82.2 \mathrm{a}$ & $88.5 \mathrm{~b}$ & $87.6 \mathrm{a}$ & $87.4 \mathrm{a}$ & $88.1 \mathrm{~b}$ & $84.0 \mathrm{a}$ & $89.6 \mathrm{a}$ & $89.6 \mathrm{a}$ & $88.6 \mathrm{a}$ & $92.3 \mathrm{~b}$ \\
\hline $\mathrm{T}_{2}$ & $78.2 \mathrm{a}$ & $83.2 \mathrm{a}$ & $84.8 \mathrm{~b}$ & $88.6 \mathrm{~b}$ & $88.7 \mathrm{a}$ & $87.6 \mathrm{a}$ & $87.8 \mathrm{~b}$ & $87.8 \mathrm{~b}$ & $90.6 \mathrm{a}$ & $89.1 \mathrm{a}$ & $88.3 \mathrm{a}$ & $91.8 \mathrm{~b}$ \\
\hline P-value & 0.49 & 0.86 & 0.01 & 0.01 & 0.31 & 0.53 & 0.01 & 0.01 & 0.10 & 0.82 & 0.20 & 0.01 \\
\hline $2008-09 * *$ & 78 & 84 & 91 & 98 & 105 & 112 & 119 & 131 & 138 & 153 & 166 & 180 \\
\hline $\mathrm{T}_{0}$ & $83.3 \mathrm{a}$ & $80.0 \mathrm{a}$ & $81.4 \mathrm{a}$ & $79.2 \mathrm{a}$ & $79.9 \mathrm{a}$ & $80.9 \mathrm{~b}$ & $82.9 \mathrm{a}$ & $84.0 \mathrm{a}$ & $88.7 \mathrm{a}$ & $92.3 \mathrm{a}$ & $88.6 \mathrm{a}$ & $89.6 \mathrm{a}$ \\
\hline $\mathrm{T}_{1}$ & $82.9 \mathrm{a}$ & $81.4 \mathrm{a}$ & $80.0 \mathrm{a}$ & $78.0 \mathrm{a}$ & $77.0 \mathrm{a}$ & $77.4 \mathrm{a}$ & $82.4 \mathrm{a}$ & $83.6 \mathrm{a}$ & $86.9 \mathrm{a}$ & $93.7 \mathrm{a}$ & $89.5 \mathrm{a}$ & $94.9 \mathrm{~b}$ \\
\hline $\mathrm{T}_{2}$ & $86.1 \mathrm{a}$ & $81.3 \mathrm{a}$ & $78.5 \mathrm{a}$ & $78.6 \mathrm{a}$ & $78.6 \mathrm{a}$ & $78.9 \mathrm{ab}$ & $81.7 \mathrm{a}$ & $83.3 \mathrm{a}$ & $85.8 \mathrm{a}$ & $88.5 \mathrm{a}$ & $89.9 \mathrm{a}$ & $92.5 \mathrm{ab}$ \\
\hline P-value & 0.67 & 0.53 & 0.47 & 0.80 & 0.39 & 0.12 & 0.72 & 0.85 & 0.20 & 0.26 & 0.77 & 0.05 \\
\hline 2009-10** & 87 & 94 & 101 & 108 & 115 & 122 & 129 & 136 & 143 & 150 & 162 & 175 \\
\hline$\overline{\mathrm{T}_{0}}$ & $74.0 \mathrm{a}$ & $75.8 \mathrm{a}$ & $78.3 \mathrm{a}$ & $82.0 \mathrm{~b}$ & $85.2 \mathrm{a}$ & $88.2 \mathrm{a}$ & $87.4 \mathrm{a}$ & $80.7 \mathrm{a}$ & $81.9 \mathrm{a}$ & $83.5 \mathrm{a}$ & $80.3 \mathrm{a}$ & $82.0 \mathrm{a}$ \\
\hline $\mathrm{T}_{1}$ & $73.4 \mathrm{a}$ & $74.1 \mathrm{a}$ & $78.6 \mathrm{a}$ & $85.8 \mathrm{~b}$ & $86.0 \mathrm{a}$ & $86.8 \mathrm{a}$ & $85.6 \mathrm{a}$ & $80.1 \mathrm{a}$ & $82.7 \mathrm{a}$ & $84.9 \mathrm{a}$ & $86.3 \mathrm{~b}$ & $85.8 \mathrm{~b}$ \\
\hline $\mathrm{T}_{2}$ & $75.5 \mathrm{a}$ & $75.8 \mathrm{a}$ & $78.1 \mathrm{a}$ & $81.5 \mathrm{a}$ & $85.8 \mathrm{a}$ & $87.9 \mathrm{a}$ & $86.1 \mathrm{a}$ & $81.8 \mathrm{a}$ & $83.2 \mathrm{a}$ & $82.2 \mathrm{a}$ & $83.3 \mathrm{ab}$ & $86.0 \mathrm{~b}$ \\
\hline$\overline{P \text {-value }}$ & 0.33 & 0.36 & 0.92 & 0.03 & 0.79 & 0.62 & 0.33 & 0.41 & 0.59 & 0.18 & 0.01 & 0.01 \\
\hline
\end{tabular}

Different letters within a row represent significant differences at $\mathrm{p}<0.05$; DAT $=$ days after transplant (letras distintas dentro de una fila representan diferencias significativas a $<<0,05 \mathrm{DAT}=$ días después del trasplante); *values corresponding to equatorial diameter of fruits (valores correspondientes al diámetro equatorial de frutas); **values corresponding to polar diameter of fruits (valores correspondientes a diámetro polar de frutas). 


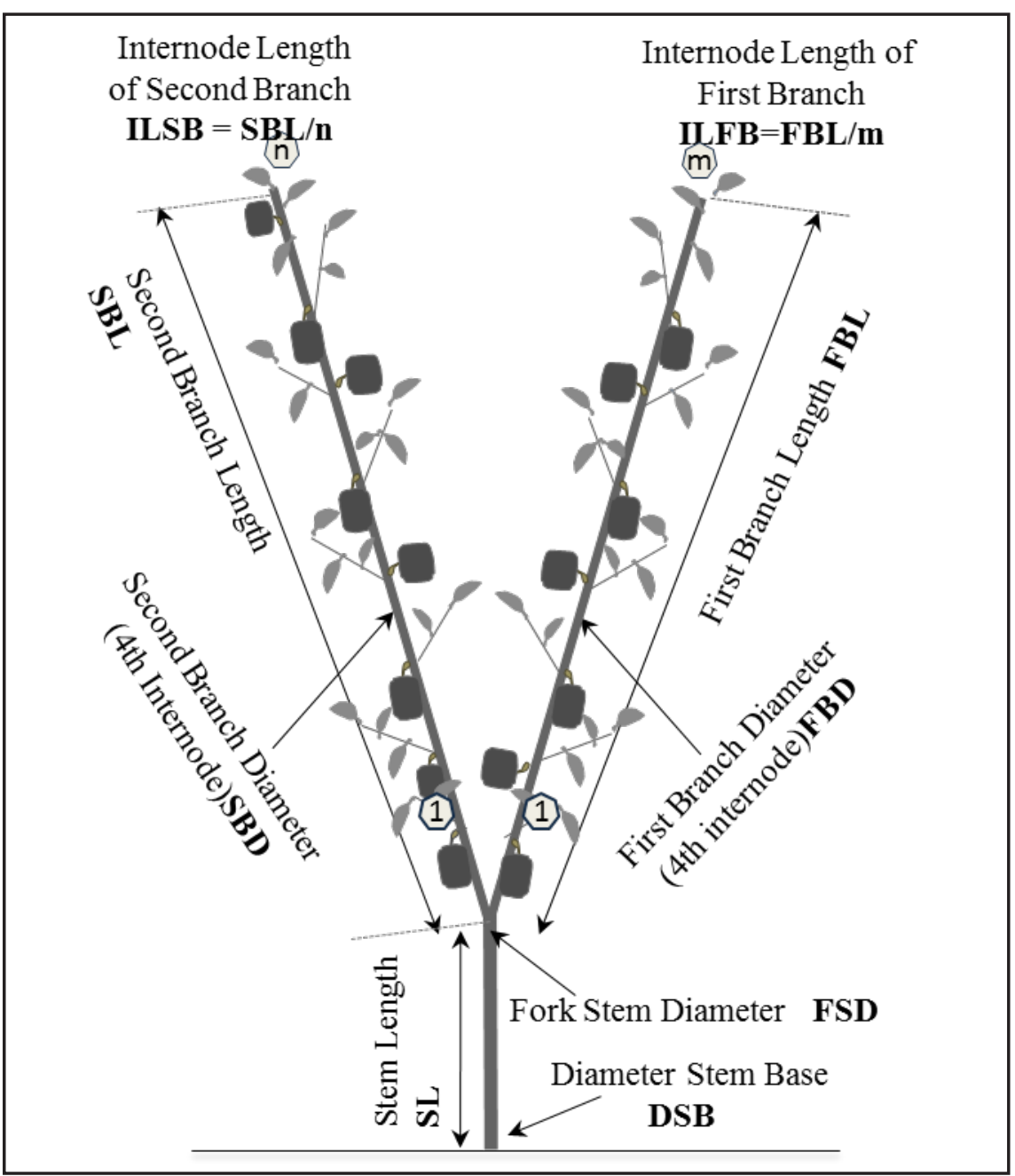

Figure 1. Morphological parameters evaluated in the pepper plant (parámetros morfológicos evaluados en la planta de pimiento). Almería (Spain), Foundation UAL, 2008-2010.

when they applied caffeine compared to the control.

Anumber of studies on the application of caffeine to a diversity of crops have shown contradictory results on plant growth, ranging from stimulation of growth to partial or complete inhibition of growth. These discrepancies may be a result of different concentrations of caffeine, different caffeine application periods, measurements taken from different plant organs, and different types of evaluations.

A number of experiments on plant organs performed in vitro by Vitória \& Mazzafera (1998) demonstrated that caffeine acts as a growth stimulator. Experiments on cotyledons of Rhapanus sativus with caffeine concentrations ranging from 0.051 to $51.00 \mu \mathrm{M}$ in $10.00-\mu \mathrm{M}$ intervals (for 72 hours of exposure) resulted in longer cotyledons when compared to the control at all concentrations used. In experiments on stem sections of Glycine max using concentrations between 0.1 and 1,000 $\mu \mathrm{M}$ (during 48 hours of exposure), concentrations between $1 \mu \mathrm{M}$ and 1 $\mathrm{mM}$ resulted in increased stem length compared to the control. In cotyledons of Cucumis sativus exposed to caffeine concentrations between 0.051 and 51.00 $\mu \mathrm{M}$ for 16 hours, more chlorophyll accumulated compared to control at all the concentrations used.

Experiments conducted on hypocotyl cuttings of Phaseolus aureus exposed to caffeine concentrations of 50, 100, 200, $500,1,000$ and $2,000 \mu \mathrm{M}$ for a week resulted in a $26.8 \%$ inhibition in number of roots compared to the control. At 50 $\mu \mathrm{M}$, an inhibition of the root length was observed in $41.7 \%$ of cases. A total inhibition of root production at 2,000 $\mu \mathrm{M}$ was observed (Batish et al., 2008).

Experiments where roots of Pisum sativum seedlings were immersed (24$26 \mathrm{~mm}$ long) in caffeine concentrations of $1,000,10,000$ and $25,000 \mu \mathrm{M}$ for 3 min and then evaluated 24 hours later did not show differences in root length. However, significant reductions of 28 and $51 \%$ in number of root cells were observed at concentrations of 10,000 and 25,000 $\mu \mathrm{M}$, respectively (CurlangoRivera et al., 2010).

Helianthus annuus seeds immersed for 24 hours in caffeine concentrations from 2,575 to $103,000 \mu \mathrm{M}$ and the displayed subsequent plants evaluated after a month, presented growth inhibition and an increase in the amount of seeds per plant at concentrations between 51,500 and 103,000 $\mu \mathrm{M}$. At concentrations between 90,000 and $103,000 \mu \mathrm{M}$, more and heavier seeds were observed (Khursheed et al., 2009). Capsicum annuum seeds immersed for 12 hours in caffeine concentrations from 2,575 to $38,660 \mu \mathrm{M}$ presented an increase of mean plant height (Kumar \& Tripathi, 2004). Seeds of Amaranthus spinosus, Echinocloa colonum, Avena fatua and Vicia sativa immersed in several caffeine concentrations for 8 hours, presented inhibited germination at concentrations of $6,180,10,300$, 25,750 and $51,500 \mu \mathrm{M}$, respectively (Rizvi et al., 1981).

To evaluate elongation of the final internode and the new growth per week, 1 and $100 \mu \mathrm{M}$ of caffeine were applied to leaves of Mucuna deeringiana and Cajanus cajan seedlings; there were no differences observed when seedlings were compared to the control (Vitória \& Mazzafera, 1998).

These studies suggest a highly variable influence of caffeine on the germination and development of the different vegetable structures. In these studies there are differences in evaluation methods, species, organs, application times, doses and evaluation times.

The methodology applied in the current paper is substantially different than the methodology used in previous studies. This experiment was conducted 
in real cultivation conditions, under a greenhouse and with fertigation applications during the entire cultivation cycle over production periods. The concentrations evaluated are within the range used by Vitória \& Mazzafera (1998) who obtained positive results for stimulation of growth in their in vitro caffeine exposure experiments. Caffeine exposure should be continuous for a maximum period of 3 days.

In the present study we concluded that caffeine doses of 2.25 and 9.00 $\mu \mathrm{M}$ applications during the entire cultivation cycle over production period neither stimulated nor inhibited the development or yield of the pepper crop.

\section{REFERENCES}

ANAYAAL; WALLER GR; OKINDA-OWUOR P; FRIEDMAN J; CHOU CH; SUZUKI T; ARROYO-ESTRADA JF; CRUZ-ORTEGA R. 2002 The Role of Caffeine in the production decline due to autotoxicity in coffee and tea plantations. In: REIGOSA M; PEDROL N. (eds). Allelopathy: from molecules to ecosystems. Enfield: Science Publishers Inc. p.71-91.

ASHIHARA H. 2006. Metabolism of alkaloids in coffee plants. Brazilian Journal of Plant Physiology 18: 1-8.

BATISH DR; KAUR M; KOHLI RK; YADAV SS. 2008. Caffeine affects adventitious rooting and causes biochemical changes in the hypocotyls cuttings of mung bean (Phaseolus aureus). Acta Physiologiae Plantarum 30: 401-405.

CROTEAUR; KUTCHAN TM; LEWIS NG. 2000. Natural products (secondary metabolites). In: BUCHANAN BB; GRUISSEM W; JONES R. (eds). Biochemistry and molecular biology of plants. Rockville: American Society of Plant
Physiologists. 1250-1318.

CURLANGO-RIVERA G; DUCLOS VD; EBOLO JJ; HAWES CM. 2010. Transient exposure of root tips to primary and secondary metabolites: Impact on root growth and production of border cells. Plant and Soil 332: 267-275.

HADACEK H. 2002. Secondary metabolites as plant traits: current assessment and future perspectives. Critical Reviews in Plant Sciences 21: 273-322.

HESSE M. 2002. Alkaloids: Nature's Curse or Blessing? Zürich: Wiley-VCH. 426p.

HITOSHI S. 2006. Cytokinins: Activity, biosynthesis, and translocation. Annual Review of Plant Biology 57: 431-449.

HOLLINGSWORTH RG; ARMSTRONG JW; CAMPBELL E. 2002. Caffeine as a repellent for slugs and snails: at high concentrations this stimulant becomes a lethal neurotoxin to garden pests. Nature 417: 915-916.

KHURSHEED T; ANSARI MYK; SHAHAB D. 2009. Studies on the effect of caffeine on growth and yield parameters in Helianthus annuus variety Modern. Biology and Medicine 1: $56-60$.

KIM YS; UEFUJI H; OGITA S; SANO H. 2006. Transgenic tobacco plants producing caffeine: a potential new strategy for insect pest control. Transgenic Research 15: 667-672.

KUMAR G \& TRIPATHI A. 2004. Mutagenic response of caffeine in Capsicum annuum Journal Indian Botanical Society 83: 136-140.

MATHAVAN S; PREMALATHA Y; CHRISTOPHER MSM. 1985. Effects of caffeine and theophylline on the fecundity of four lepidopteran species. Experimental Biology 44: 133-138.

MATSUBARA S. 1990. Structure-activity relationships of cytokinins. Critical Reviews in Plant Sciences 9: 17-57.

MOK MC. 1994. Cytokinin metabolic enzimes. In: MOK DWS; MOK MC. (eds). Cytokinins: chemistry, activity and function. Florida: Boca Raton. 155-166.

MONTES-ZAVALA O; DIÁNEZ-MARTÍNEZ F; CAMACHO-FERRE F. 2013. Effect of caffeine on grafted watermelon crop yields and fruit quality under greenhouse conditions. Journal of Food, Agriculture \& Environment 11: 784-787.

MORSUCCI R; CURVETTO N; DELMASTRO S. 1991. Involvement of cytokinin and adenosine 3,5 cyclic monophosphate in stomatal movement in Vicia faba. Plant Physiology and Biochemistry 29: 537-547.

RIZVI SJH; MUKERJI D; MATHUR SN. 1981. Selective phytotoxicity of $1,3,7$ trimethylxantine between Phaseolus mungo and some weeds. Agricultural and Biological Chemistry 45: 1255-1256.

SINGH HP; BATISH DR; KOHLI RK. 2001. Allelopathy in agroecosystems: an overview. Journal of Crop Production 4: 1-41.

SMYTH AD. 1992. Effect of methylxanthine treatment on rice seedling growth. Journal Plant Growth Regulation 11: 125-128.

TAIZ L; ZEIGER E. 2002. Plant Physiology. $3^{\text {rd }}$ ed. Massachusetts: Sinaver Associates.

TAO GQ; LETHAM DS; HOCART CH; SUMMONS RE. 1991. Inhibitors of Cytokinin Metabolism III. The Inhibition of Cytokinin $\mathrm{N}$-Glucosylation in Radish Cotyledons. Journal Plant Growth Regulation 10: 179-185.

TRUTA E; ZAMFIRACHE MM; OLTEANU Z. 2011. Caffeine induced genotoxic effects in Phaseolus vulgaris and Raphanus sativus. Botanica Serbica 35: 49-54.

UEFUJI H; OGITA S; YAMAGUCHI Y; KOIZUMI M; SANO H. 2003. Molecular cloning and functional characterization of three distinct $\mathrm{N}$-methyltranferases involved in the caffeine biosynthetic pathway in coffee plants. Plant Physiology 132: 372-380.

VITÓRIAAP; MAZZAFERAP. 1998. Cytokininlike effects of caffeine in bioassays. Biologia Plantarum 40: 329-335.

WINK M. 2004. Allelochemical properties of quinolizidine alkaloids. In: MACIAS FA; GARCÍA-GALINDO JC; MOLINILLO JMG; CUTLER HG. (eds) Allelopathy-chemistry and made of action of allelochemicals. Florida: Boca Ratón. 183-200. 